\title{
Electroacupuncture Ameliorates Depressive-Like Behaviors in Poststroke Rats via Activating the tPA/BDNF/TrkB Pathway
}

This article was published in the following Dove Press journal:

Neuropsychiatric Disease and Treatment

\author{
Hao Dong ${ }^{1, *}$ \\ Yan-Qiang Qin ${ }^{2, *}$ \\ Ying-Chun Sun ${ }^{2, *}$ \\ Hai-Jiang Yao ${ }^{2}$ \\ Xian-Kuan Cheng ${ }^{2}$ \\ Yan Yu' \\ Shou-Si Lu' \\ 'Beijing Bo'ai Hospital, China \\ Rehabilitation Research Center, China \\ Rehabilitation Science Institute, Beijing, \\ People's Republic of China; ${ }^{2}$ Treatment \\ Center of TCM, Beijing Bo'ai Hospital, \\ China Rehabilitation Research Center, \\ China Rehabilitation Science Institute, \\ School of Rehabilitation Medicine, Capital \\ Medical University, Beijing, People's \\ Republic of China \\ *These authors contributed equally to \\ this work
}

Background: Electroacupuncture (EA) is a form of physical therapy that has been widely used in clinical practice in China. Post-stroke depression (PSD) is the most common neuropsychiatric complication after stroke. EA has been shown to have beneficial effects on PSD patients. However, the potential mechanism underlying the protective effects of EA on PSD remains unclear. Here, we investigated whether tissue plasminogen activator (tPA)/ brain-derived neurotrophic factor (BDNF)/tyrosine kinase receptor B (TrkB) signaling pathway participates in the therapeutic effects of EA in a rat PSD model.

Methods: Experimental PSD was induced by combining middle cerebral artery occlusion (MCAO) with chronic unpredictable mild stimulation (CUMS) in adult male rats. Bodyweight gain, neurological score, sucrose preference, and open field test were determined at $0,7,14$, and 35 days after completing MCAO. The protein expressions of tPA, precursor BDNF (proBDNF), mature BDNF (mBDNF), and TrkB were measured by immunofluorescence and Western blot analysis. The tPA inhibitor plasminogen inhibitor-1 (PAI-1) was used to explore whether tPA plays a crucial role in the protective effects of EA on PSD.

Results: Compared with the sham rats, the PSD rats showed decreased bodyweight, deteriorated neurological score, and significant depressive-like behaviors. EA remarkably reversed bodyweight loss, neurological deficit, and depressive-like behaviors in PSD rats. Immunofluorescence staining and Western blot analysis showed that PSD-induced decreased expression of tPA, mBDNF, and TrkB were prevented by EA. Furthermore, we found that the effects of EA against PSD-induced depressive-like behaviors were abolished by PAI-1, the specific inhibitor of PAA. Conclusion: Our results suggest that the improvement in depressive-like behaviors induced by EA is likely achieved via activation of the tPA/BDNF/TrkB pathway.

Keywords: electroacupuncture, poststroke depression, depressive-like behaviors, tPA/ $\mathrm{BDNF} / \mathrm{TrkB}$, chronic unpredictable mild stimulation

\section{Introduction}

Cerebral stroke has been considered as a major health concern. By 2050 a growing number of people around the world will be aged 65 years or older which would lead to an increase in age-related diseases including the stroke. ${ }^{1}$ Post-stroke depression (PSD) is considered the most common neuropsychiatric consequence following a stroke. The estimated prevalence of PSD is suggested to be $30-50 \%$ among stroke survivors. ${ }^{2}$ This disorder is closely associated with increased disability and lowered quality of life, which places a heavy burden on health care systems, families, and society. ${ }^{3}$ Selective serotonin reuptake inhibitors (SSRIs) are currently
Correspondence: Yan-Qiang Qin; Ying-Chun Sun

Treatment Center of TCM, Beijing Bo'ai Hospital, China Rehabilitation Research Center, China Rehabilitation Science Institute, No. 10 Jiaomen North Road, Beijing, 100068, People's Republic of China Tel +86 I86I225I24I

Email yanqiangqin@I26.com; syccrrc@sina. com
Neuropsychiatric Disease and Treatment 2021:17 1057-1067

1057

DovePress if in $>$ 
considered as the standard treatment for PSD because of their proven efficacy. ${ }^{4}$ However, SSRI administration raises the risk of cerebral hemorrhage and other side effects. $^{5}$

It has been suggested that brain-derived neurotrophic factor (BDNF) plays an important role in the pathophysiological mechanisms of PSD. ${ }^{6}$ Decreased concentration of serum BDNF has been demonstrated in patients with PSD. $^{7}$ The unbalance between BDNF and precursor BDNF (proBDNF) in the brain has also been found in the PSD rats. ${ }^{8}$ Besides, tissue plasminogen activator (tPA) is an important factor that affects the balance of BDNF/proBDNF through activating the extracellular protease plasmin. ${ }^{9}$ Tyrosine receptor kinase B (TrkB), the receptor of $\mathrm{BDNF}$, is another vital signaling molecule involved in the pathogenesis of PSD. ${ }^{10}$ Current evidence suggests the $\mathrm{tPA} / \mathrm{BDNF} / \mathrm{TrkB}$ signaling pathway maybe participate in the occurrence and development of PSD. ${ }^{9,11}$

Electroacupuncture (EA) has been used to treat a wide variety of medical conditions, such as knee osteoarthritis, ${ }^{12}$ stress urinary incontinence, ${ }^{13}$ functional constipation, ${ }^{14}$ and so on. Recently, EA has been demonstrated to have beneficial effects on neuropsychiatric disorders, ${ }^{15-18}$ including PSD. ${ }^{19}$ Animal studies showed that hippocampal sonic hedgehogsignaling pathway, ${ }^{20}$ Keap1/Nrf2 antioxidant signaling pathway, $^{21}$ and endocannabinoid signaling pathway ${ }^{22}$ may be involved in the protective mechanism of EA on PSD. What remains unclear, however, is whether the tPA/BDNF/ TrkB signaling pathway participates in the protective effects of EA on PSD.

In the present study, we examined whether EA could attenuate depressive-like behaviors in a rat model of PSD. We also determined whether the tPA/BDNF/TrkB signaling pathway was involved in the protective effects of EA. Results of this study will improve our understanding of the mechanism underlying EA on PSD.

\section{Materials and Methods}

\section{Animals}

Sixty male Sprague-Dawley rats weighing 180-220 g were obtained from Vital River Laboratories (Beijing, China). The temperature was maintained at $23 \pm 2{ }^{\circ} \mathrm{C}$ and the relative humidity was controlled at $40 \%-70 \%$. Water and standard chow were available ad libitum throughout the study. All animal experiments were performed according to the ARRIVE guidelines, American Association for Accreditation of Laboratory Animal Care's policy, and
Guide for the Care and Use of Laboratory Animals issued by the Institute for Laboratory Animal Research. All of the experimental protocols were approved by the Experimental Ethics Committee of China Rehabilitation Research Center (No. 20180433).

\section{Experimental Design \\ Experiment I}

To observe the effects of EA on behaviors of PSD rats, bodyweight gain, neurological score, and depressive-like behaviors were determined at $0,7,14$, and 35 days after completing middle cerebral artery occlusion (MCAO). Rats were randomly assigned to sham, model, fluoxetine, and EA groups ( $\mathrm{n}=10$ per group), as shown in Figure 1. Rats in the model group were subjected to MCAO combined with chronic unpredictable mild stimulation (CUMS). Rats in the sham group were subjected to experimental procedures similar to the model group except for MCAO and chronic stress. Rats in the fluoxetine group were subjected to MCAO, CMUS, and fluoxetine treatment (Prozac, $0.2 \mathrm{mg} / \mathrm{kg}$, Eli Lilly \& Co.). Rats in the EA group were subjected to MCAO, CMUS, and EA intervention. To investigate whether the $\mathrm{tPA} / \mathrm{BDNF} / \mathrm{TrkB}$ pathway was involved in this process, the rats were sacrificed at 35 days and the expressions of tPA, proBDNF, mature BDNF (mBDNF), and TrkB were detected.

\section{Experiment II}

To identify the role of tPA activation in the protective effect of EA intervention, we used the tPA-specific inhibitor PAI-1 in the experiment. Rats were randomly divided into two groups: Model+PAI-1 and Model+EA+PAI-1 $(\mathrm{n}=10$ per group). PAI-1 was injected intracerebroventricularly at a dose of $1.9 \mathrm{ug} / 10 \mathrm{ul} / \mathrm{rat} / \mathrm{day}$.

\section{Post-Stroke Depression Model}

The post-stroke depression (PSD) model was induced by combining MCAO with CMUS. MCAO was established by using modifying Longa's method. ${ }^{23}$ In brief, rats were anesthetized with $10 \%$ chloral hydrate intraperitoneally at a dose of $300 \mathrm{mg} / \mathrm{kg}$. A midline incision was made in the neck and the left common carotid artery was isolated. Subsequently, a monofilament nylon suture was inserted into the external carotid artery and advanced to occlude the middle cerebral artery. The filament was left in the artery for $90 \mathrm{~min}$ and then withdrawn for reperfusion. Successful MCAO was confirmed by using a 5-point neurological score. CUMS was arranged for 4 consecutive 
Sham
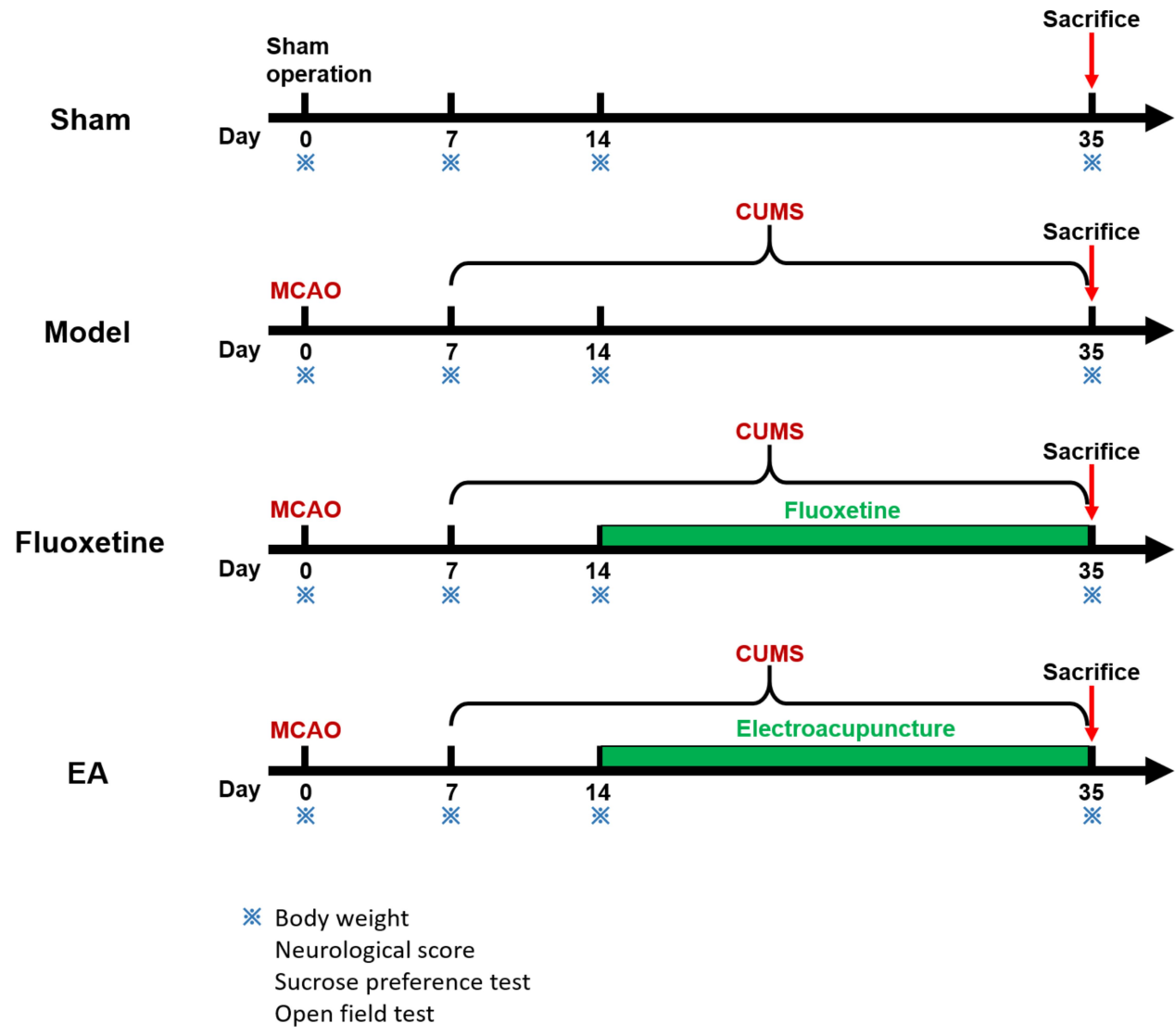

Figure I Schematic showing the timeline of the intervention and behavioral tests.

weeks with one stressor each day after 1 week of recovery from the MCAO surgery. The stressors included deprivation of food and water for 24 hours, $45^{\circ}$ cage tilt for 24 hours, humid environment for 24 hours, day and night reversed for 24 hours, wrap restraint for 2 hours, vibration for $30 \mathrm{~min}$, and tail clamping for 1 minute.

\section{EA Treatment}

Fourteen days after MCAO, rats in the EA group received EA treatment for 3 weeks. Briefly, rats were maintained on a platform and the acupoints Baihui (GV20), Yintang (GV29), Zhongwan (CV12), Guanyuan (CV4) were stimulated by acupuncture needles for $30 \mathrm{~min}$ daily. The acupuncture needles $(0.16 \times 7 \mathrm{~mm})$ were inserted at a depth of $4 \mathrm{~mm}$ into these acupoints. Needles were then connected to the HANS200A Acupuncture Point Nerve Stimulator (Nanjing, China) with a frequency of $2 \mathrm{~Hz}$. The electrical stimulus intensity was set at the threshold for a detectable muscle twitch (1 mA).

\section{Neurological Score}

The neurological score was performed at $0,7,14$, and 35 days after MCAO to evaluate the neurological deficit according to previous publications. Neurological status was graded according to a 5-point grading system: 0, no observable deficit; 1 , forelimb flexion; 2 , unidirectional circling; 3 , falling to the contralateral injury side; 4 , unable to walk spontaneously or lack of consciousness.

\section{Sucrose Preference Test}

The sucrose preference test was performed at $0,7,14$, and 35 days after MCAO to measure the depressive-like behavior. Rats were given access to two preweighted bottles, one containing tap water and the other containing sucrose solution (1\%). The consumption of sucrose solution was quantified by weighing the bottles. Sucrose preference was calculated using the following formula: sucrose preference $(\%)=$ sucrose intake/total fluid intake $\times 100$.

\section{Open Field Test}

The open field test was performed at $0,7,14$, and 35 days after MCAO to assess general locomotor activity and anxiety-related behavior. Rats were individually placed in the center of a cube $(100 \mathrm{~cm} \times 100 \mathrm{~cm} \times 100 \mathrm{~cm})$ for 10 minutes. Total distance traveled (grid crossing) and 
vertical activity of rats were analyzed. The apparatus was cleaned with $75 \%$ ethanol between tests.

\section{Enzyme-Linked Immunosorbent Assay}

We used commercial enzyme-linked immunosorbent assay kits (RayBiotech, US) to measure the levels of BDNF and proBDNF in serum. Blood was collected at the time of sacrifice and the blood sample was centrifuged to separate

A

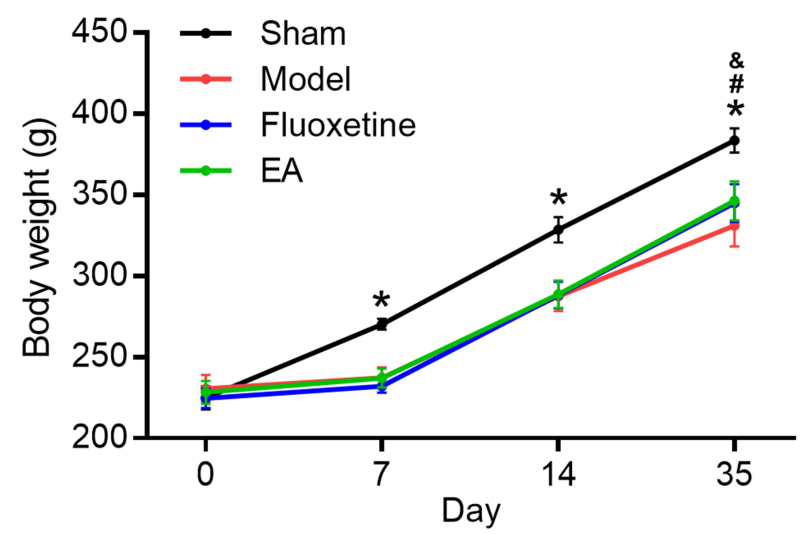

C

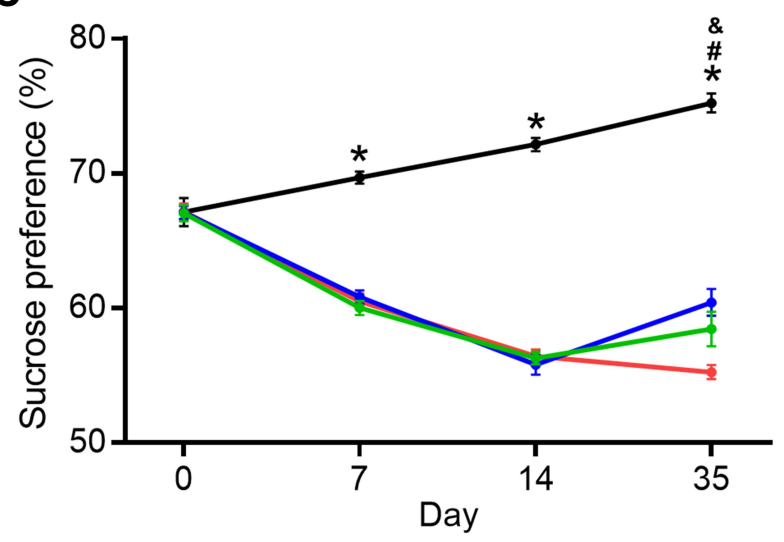

E

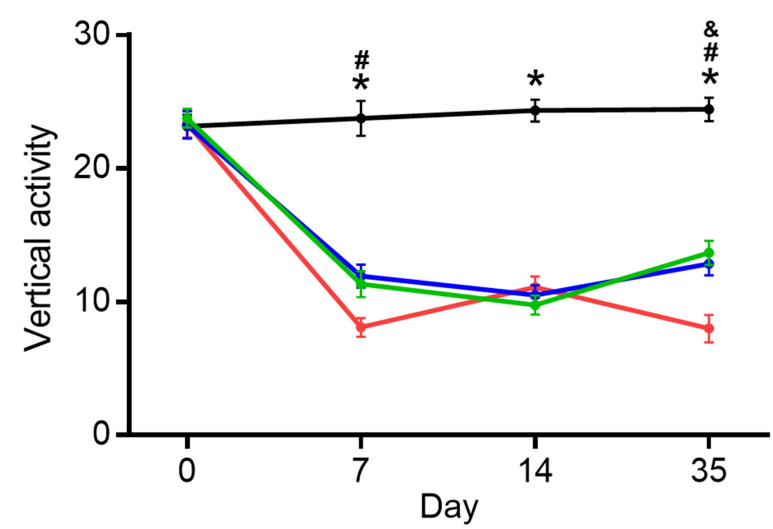

the serum. All serum samples were stored at $-80^{\circ} \mathrm{C}$ until use. Analyses were performed according to the manufacturers' protocol.

\section{Western Blot}

Immediately after sacrificing the rats, brains were rapidly dissected on ice and the prefrontal cortex was isolated. The protein concentration was determined by a BCA protein assay

B
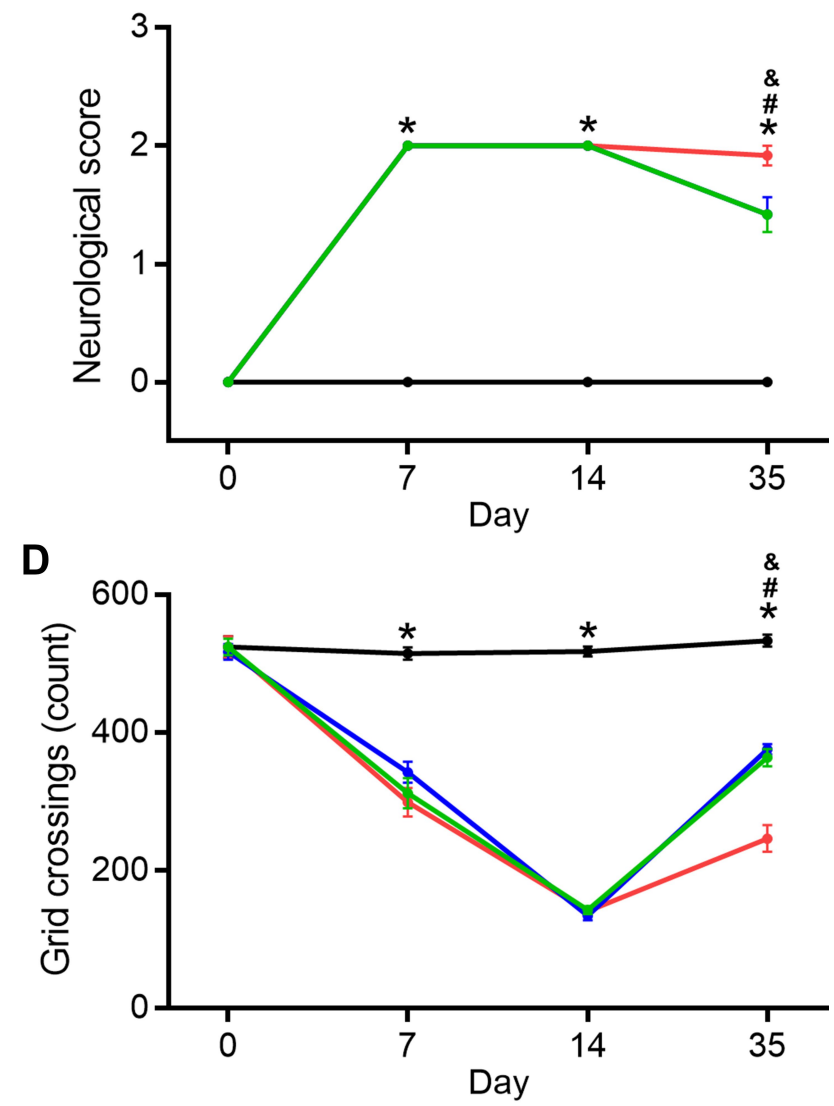

Figure 2 The effect of EA on depressive-like behaviors in PSD rats. Bodyweight gain (A), neurological score (B), sucrose preference (C), and open field test (D and E) were determined at $0,7,14$, and 35 days after completing MCAO. ${ }^{*} \mathrm{P}<0.05$, sham vs model, ${ }^{\#} \mathrm{P}<0.05$, model vs fluoxetine, ${ }^{\circledR} \mathrm{P}<0.05$, model vs EA. 
kit. Proteins were separated by SDS-PAGE and transferred to a PVDF membrane (Millipore, USA) by electroblotting. The membranes were incubated with primary antibodies (antiBDNF, 66292-1-Ig, 1:1000, Proteintech; anti-proBDNF, p1374, 1:1000, Sigma; anti-tPA, 10147-1-AP, 1:2000, Proteintech; anti-TrkB, 13129-1-AP, 1:1000, Proteintech; antiGAPDH, ab8245, 1:5000, Abcam) overnight at $4^{\circ} \mathrm{C}$ and then with a secondary antibody for an hour at $37^{\circ} \mathrm{C}$. The immunoreactive bands were visualized using the Electro-ChemiLuminescence (ECL) kit and quantification analyses were performed using Image-Pro Plus software.

\section{Immunofluorescence}

Rats were deeply anesthetized and perfused transcardially with saline followed by $4 \%$ paraformaldehyde. Brains were removed and post-fixed in $4 \%$ paraformaldehyde overnight. After fixation, brains were dehydrated through a graded ethanol series and embedded in paraffin. Coronal brain sections were cut into $6 \mu \mathrm{m}$ thicknesses with a rotary microtome (Leica RM2235, Germany). The sections were blocked with 5\% bovine serum albumin (BSA) and incubated with primary antibodies (anti-BDNF, 1:200, Proteintech; anti-proBDNF, 1:400, Proteintech; anti-tPA, 1:200, Proteintech; antiTrkB, 1:500, Proteintech;) overnight at $4^{\circ} \mathrm{C}$ and then with a secondary antibody for $30 \mathrm{~min}$ at room temperature. Slides were visualized under a fluorescence microscope and analyzed by a researcher who was blind to the assignment group.

\section{Statistical Analyses}

All results are expressed as mean \pm SEM. The normal distribution of data was evaluated using the AndersonDarling test. The data of bodyweight, neurological score, sucrose preference, and open field test were analyzed using the two-way repeated measures ANOVA. The other data were statistically evaluated using a one-way ANOVA. A value of $\mathrm{P}<0.05$ was considered statistically significant.

\section{Results}

\section{EA Ameliorated Depressive-Like Behaviors in PSD Rats}

First, we determined the effects of EA intervention on depressive-like behaviors in PSD rats. Bodyweight loss, neurological deficit score, sucrose preference test, and open field test were evaluated at $0,7,14$, and 35 days after MCAO (Figure 2). Compared with the sham group, bodyweight in the model group was significantly decreased. But both fluoxetine and EA partly reversed
A

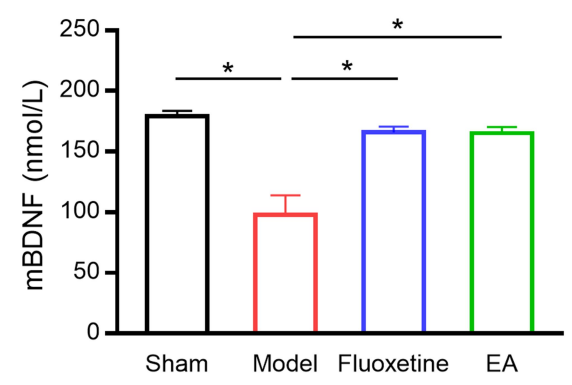

B

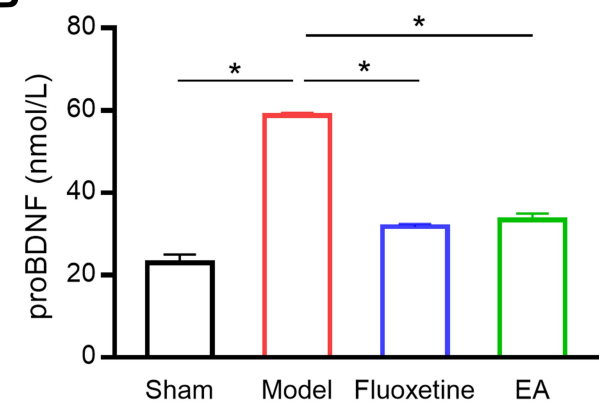

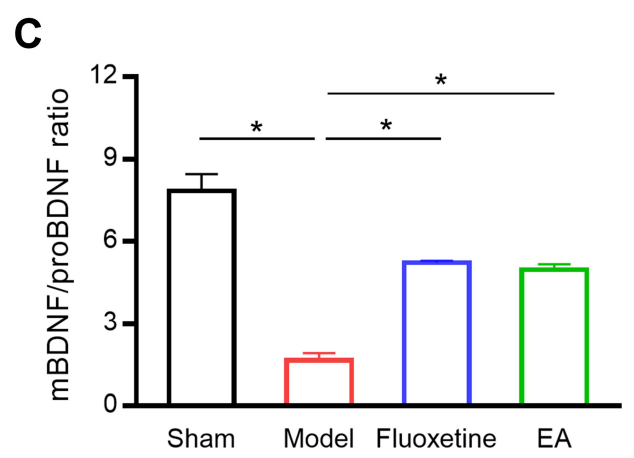

Figure 3 The effect of EA on BDNF level in the serum of PSD rats. Serum mBDNF $(\mathbf{A})$, proBDNF $(\mathbf{B})$, and $\mathrm{mBDNF} / \mathrm{proBDNF}$ ratio $(\mathbf{C})$ were detected by ELISA. $* \mathrm{P}<0.05$, compared as indicated. 
the reduction at 35 days after MCAO. Neurological score in the model group was deteriorated compared with the sham group. This deterioration was also reversed by both fluoxetine and EA intervention. In the sucrose preference test, sucrose preference of PSD rats decreased distinctly. Both fluoxetine and EA treatment alleviated depressivelike behaviors in PSD rats. In the open field test, PSD rats showed decreased grid crossing and vertical activity compared with sham rats. Rats in the fluoxetine and EA group showed significantly more grid crossing and vertical activity than rats in the model group. These results confirmed EA exerted anti-depressive effects in a rat model of PSD.

\section{EA Increased BDNF Level in the Serum of PSD Rats}

Next, we examined BDNF concentration in the serum of rats (Figure 3). Compared with the sham group, mBDNF concentration in the serum was significantly decreased in the model group. EA intervention markedly reversed the reduction of BDNF serum concentration. On the contrary, proBDNF concentration in the serum was significantly increased in the model group compared with the sham group. The increase of proBDNF serum concentration was reversed by EA treatment. Besides, the $\mathrm{mBDNF} / \mathrm{proBDNF}$ ratio was significantly decreased in PSD rats compared with sham rats. But EA intervention recovered the balance between $\mathrm{mBDNF}$ and proBDNF.

\section{EA Increased BDNF Expression in the Prefrontal Cortex of PSD Rats}

Then the expressions of $\mathrm{mBDNF}$ and proBDNF in the prefrontal cortex were further determined by Western blot and immunofluorescence staining (Figures 4 and $\underline{\mathrm{S} 1}$ ). Compared with the sham group, the expression of mBDNF was

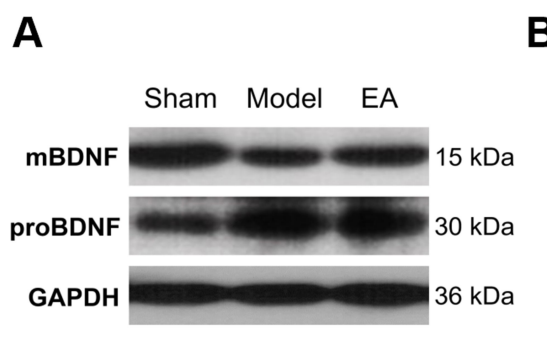

D

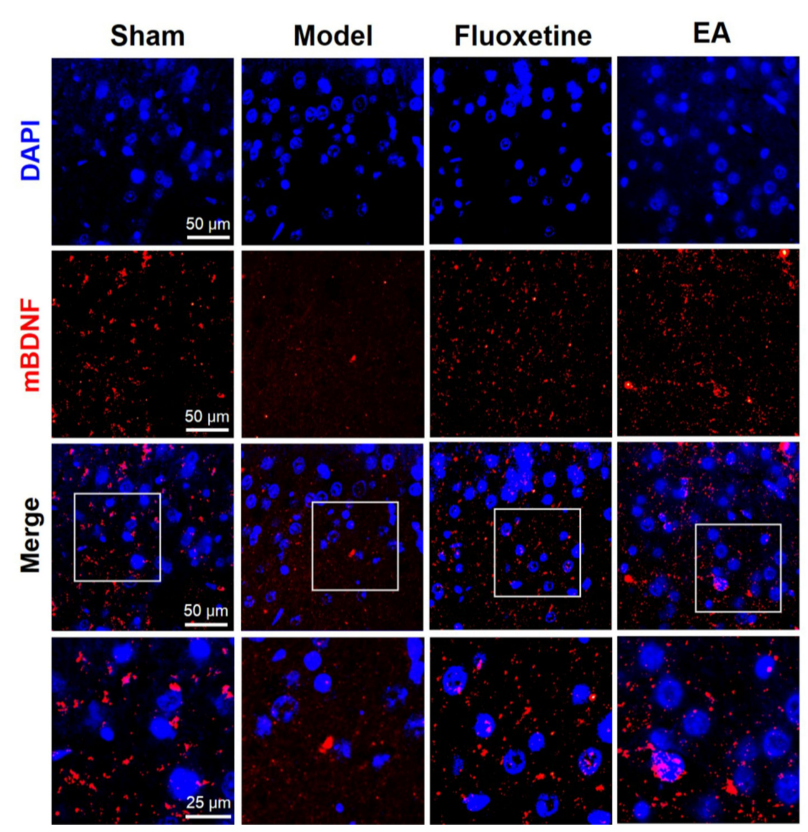

B

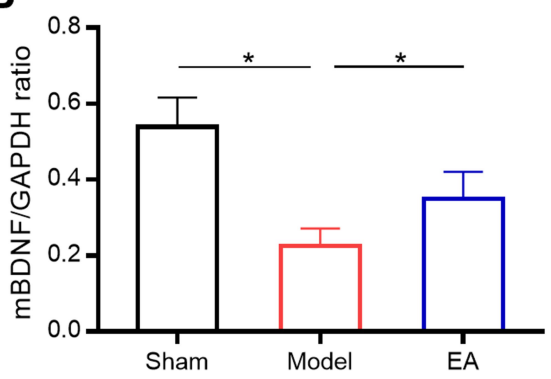

C

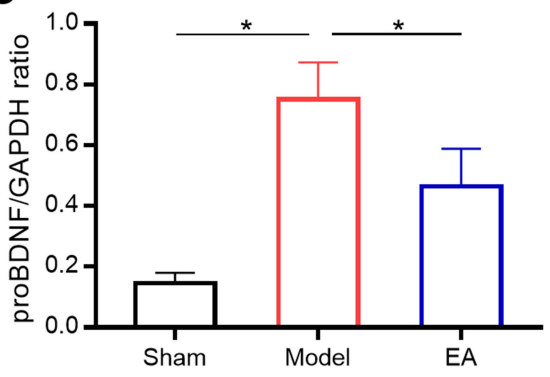

E

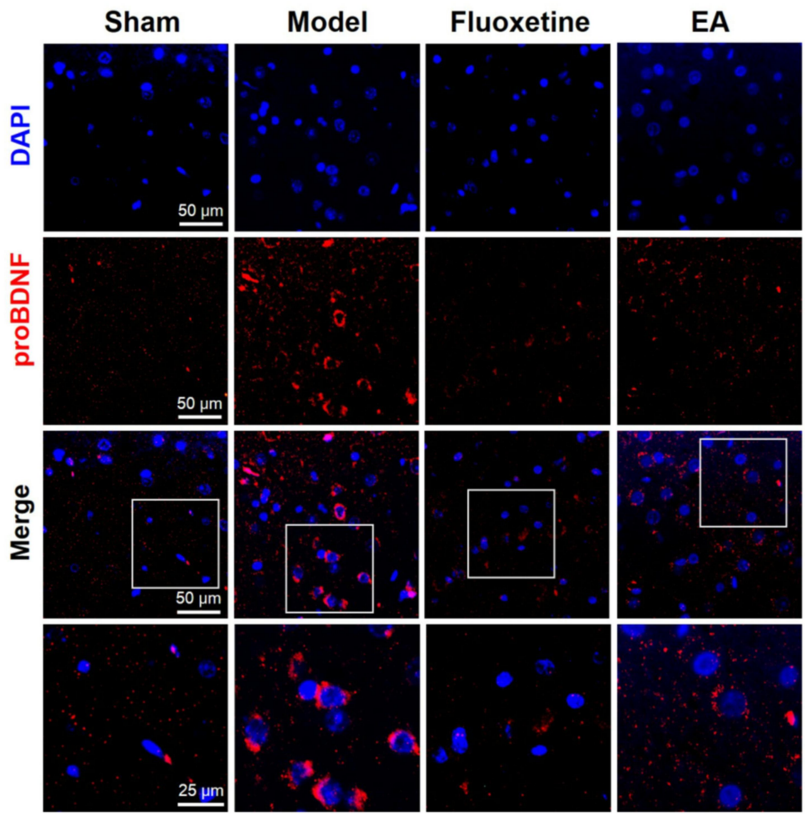

Figure 4 The effect of EA on mBDNF and proBDNF expressions in the prefrontal cortex of PSD rats. Representative immunoblots (A) and densitometry analysis of $\mathrm{mBDNF}$ and proBDNF (B and $\mathbf{C}$ ) expressions in the prefrontal cortex. Representative images of immunofluorescent staining of mBDNF and proBDNF in the prefrontal cortex ( $\mathbf{D}$ and $\mathbf{E})$. $* \mathrm{P}<0.05$, compared as indicated. 
significantly decreased in the model group. But EA treatment prevented PSD-induced reduction of $\mathrm{mBDNF}$ expression. On the other hand, a significantly higher level of proBDNF expression was observed in PSD rats than that in sham rats. The increase of proBDNF expression in PSD rats was markedly reversed by EA intervention. These results were simultaneously validated by Western blot and immunofluorescence staining.

\section{EA Increased tPA and TrkB Expressions in the Prefrontal Cortex of PSD Rats}

Subsequently, we evaluated the expressions of tPA and TrkB in the prefrontal cortex of PSD rats (Figures 5 and S1). Both Western blot and immunofluorescence staining indicated that the expressions of $\mathrm{tPA}$ and $\mathrm{TrkB}$ in the prefrontal cortex were significantly lower in the model group than that in the sham group. Interestingly, the expressions of tPA and $\operatorname{TrkB}$ were increased in the EA group compared with that in the model group. These results suggested that the tPA/BDNF/TrkB signaling pathway in the prefrontal cortex maybe participate in the protective effects of EA on PSD.

\section{PAl-I Blocked EA-Induced Improvement in PSD Rats}

To explore whether the protective effects induced by EA were dependent on the activation of tPA pathway, we injected the tPA inhibitor plasminogen inhibitor-1 (PAI-1) in EA-treated rats (Figures 6 and S2). Compared with the Model+EA group, bodyweight in both the Model+PAI-1group and Model+PAI+EA group was significantly decreased. Similarly, neurological scores in Model+PAI-1group and Model+PAI +EA group were markedly deteriorated compared with the Model+EA group. In the sucrose preference test, sucrose preference in Model+PAI-1group and Model+PAI+EA group was distinctly decreased compared with the Model+EA group. In the open field test, rats in Model+PAI-1 group and Model+PAI+EA group showed significantly decreased grid crossing and vertical activity compared with rats in the Model+EA group. All these results suggested the improvement induced by EA on depressive-like behaviors in PSD rats was dependent on the activation of tPA pathway.
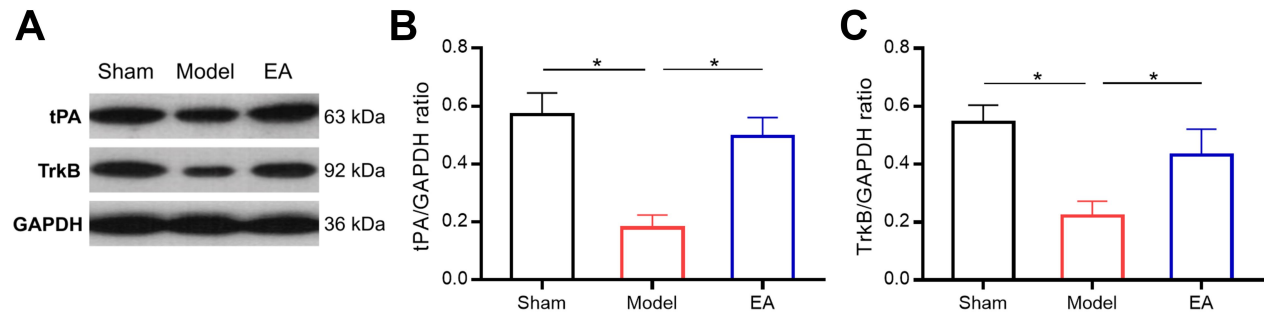

D

$\mathbf{E}$
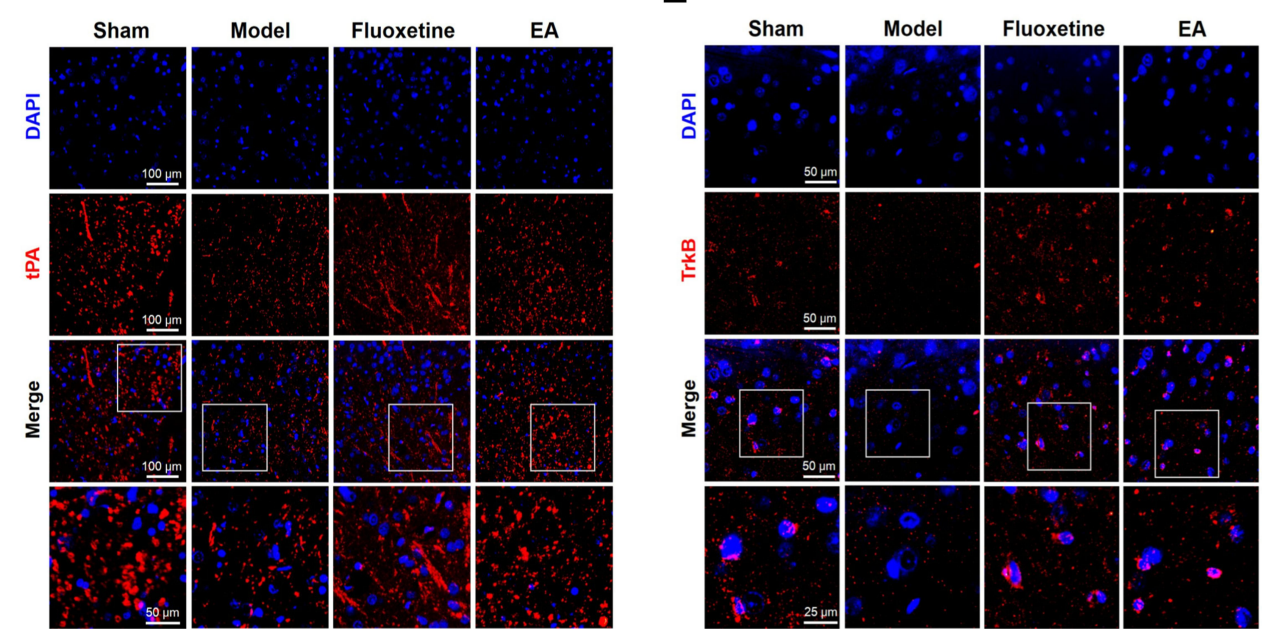

Figure 5 The effect of EA on tPA and TrkB expressions in the prefrontal cortex of PSD rats. Representative immunoblots (A) and densitometry analysis of tPA and TrkB (B and $\mathbf{C})$ expressions in the prefrontal cortex. Representative images of immunofluorescent staining of tPA and TrkB in the prefrontal cortex ( $\mathbf{D}$ and $\mathbf{E})$. $* \mathrm{P}<0.05$, compared as indicated. 
A

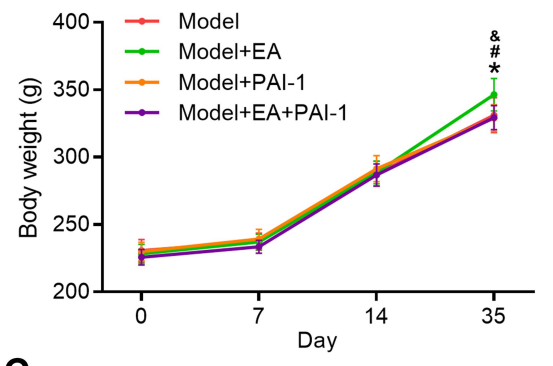

C

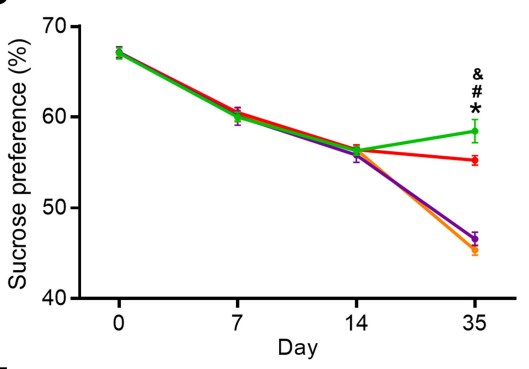

$\mathrm{E}$

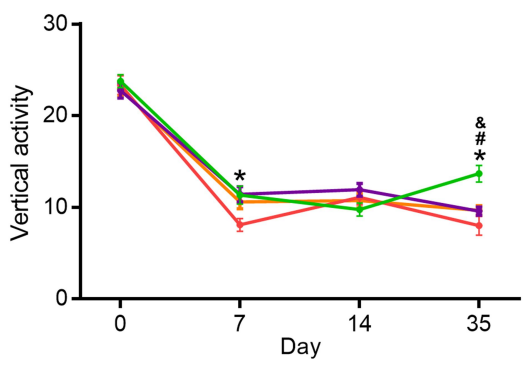

B

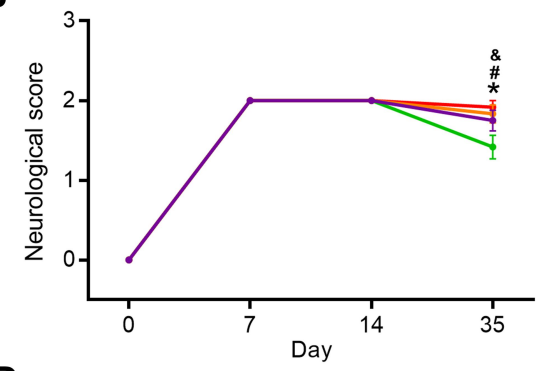

D

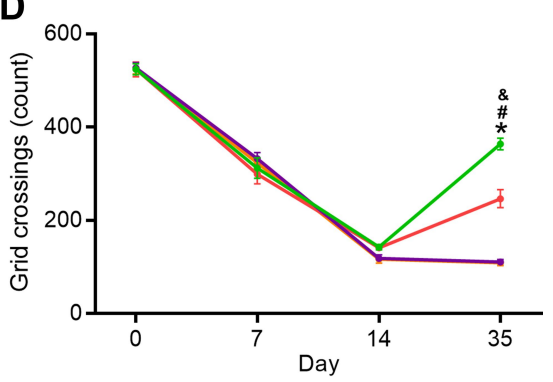

Figure 6 The effect of tPA inhibitor PAI-I on the EA-induced behavior improvement. Bodyweight gain (A), neurological score (B), sucrose preference (C), and open field test (D and E) were determined at $0,7,14$, and 35 days after completing $\mathrm{MCAO}$. ${ }^{*} \mathrm{P}<0.05$, model vs model+EA, ${ }^{\#} \mathrm{P}<0.05$, model+EA vs model+PAI-I, ${ }^{\&} \mathrm{P}<0.05$, model +EA vs model+EA+PAI-I.

\section{Discussion}

To the best of our knowledge, this is the first study that investigated whether the $\mathrm{PA} / \mathrm{BDNF} / \mathrm{TrkB}$ pathway participates in the protective effects of EA in a PSD model. In this study, we found that EA attenuated depressive-like behaviors in a PSD model. The therapeutic effects may be associated with activation of the $\mathrm{PA} / \mathrm{BDNF} / \mathrm{TrkB}$ pathway in the prefrontal cortex. Moreover, the tPA inhibitor PAI-1 significantly abolished the benefits induced by EA intervention, supporting this contention.

Depression may lead to appetite loss, which can result in weight loss. ${ }^{24}$ In our study, the combination of MCAO and CUMS caused weight loss in rats, which is consistent with previous studies. ${ }^{25}$ EA intervention as well as fluoxetine ameliorated the loss of weight. The neurological score is commonly used to quantify the extent of neurological injury. Our results showed that EA exerted a neuroprotective effect in a rat model of PSD. Interestingly, we found that the antidepressant fluoxetine also attenuated the neurological deficit in the PSD model. This is probably because fluoxetine may afford neuroprotection in the postischemic brain, and the neuroprotection might be associated with anti-inflammatory effects and anti-apoptotic effects of fluoxetine. ${ }^{26,27}$ The sucrose preference and open field test are widely accepted behavioral testing tools for assessing the antidepressant-like effects of agents. ${ }^{3}$ A decrease in sucrose intake is used as an indicator of anhedonia and the open field test measures the locomotor activity and level of anxiety. ${ }^{28}$ In our study, PSD rats showed a reduction in sucrose intake and a decrease in grid crossing and vertical activity in the open field test. Both EA and fluoxetine reversed the 
reduction, which demonstrated that the antidepressant effects of EA are similar to the effects of fluoxetine. The classical antidepressant fluoxetine was used as positive control drug in the behavioral experiments because fluoxetine is considered as the first-line treatment for PSD. ${ }^{4}$

Studies have shown that BDNF plays an important role in the pathogenesis of PSD. ${ }^{29}$ Clinical studies have revealed that patients with PSD have a lower level of serum $\mathrm{BDNF}^{30}$ and ischemic stroke patients with low serum BDNF have a much higher risk for PSD. ${ }^{31}$ In this study, PSD rats revealed a significant reduction in serum level of BDNF. Which demonstrated that the animal model of PSD used in this study, at least in part, mimics the actual clinical situation. Furthermore, our study showed that EA intervention reversed the reduction of serum BDNF caused by PSD. ProBDNF, the precursor protein of the mature form BDNF, could be converted to BDNF by extracellular proteases. We found that the serum level of proBDNF was significantly higher in PSD rats. As a result, the ratio of mBDNF to proBDNF was significantly lower in PSD rats. But EA intervention increased the serum level of proBDNF and restored the balance between mBDNF and proBDNF. All these results demonstrated that the protective effects of EA on PSD are associated with the increase of BDNF in serum.

Hippocampus and prefrontal cortex both have important roles in the pathogenic mechanism of PSD. ${ }^{32,33}$ In this study, we mainly focused on the mechanism involved in the prefrontal cortex. The mechanism involved in the hippocampus will be investigated further in our later research. Therefore, we then determined the protein expression of mBDNF and proBDNF in the prefrontal cortex. As expected, the unbalance between mBDNF and proBDNF was also found in the prefrontal cortex of PSD rats. Similarly, EA intervention restored the balance between $\mathrm{mBDNF}$ and proBDNF. The proBDNF is converted to mBDNF by tPA through activating the extracellular protease plasmin. ${ }^{34} \mathrm{BDNF}$ then binds to and activates its cognate receptor TrkB to modulate the activity of neurons. ${ }^{35}$ So the protein expressions of tPA and TrkB in the prefrontal cortex were also detected. PSD caused a significant reduction in expressions of tPA and TrkB in rats, whereas EA intervention reversed the reduction. Taken together, the current results suggest that the protective effects of EA on PSD were associated with activation of the $\mathrm{tPA} / \mathrm{BDNF} / \mathrm{TrkB}$ pathway in the prefrontal cortex. Furthermore, our results also showed that the therapeutic action of EA on depressive-like behaviors in PSD rats

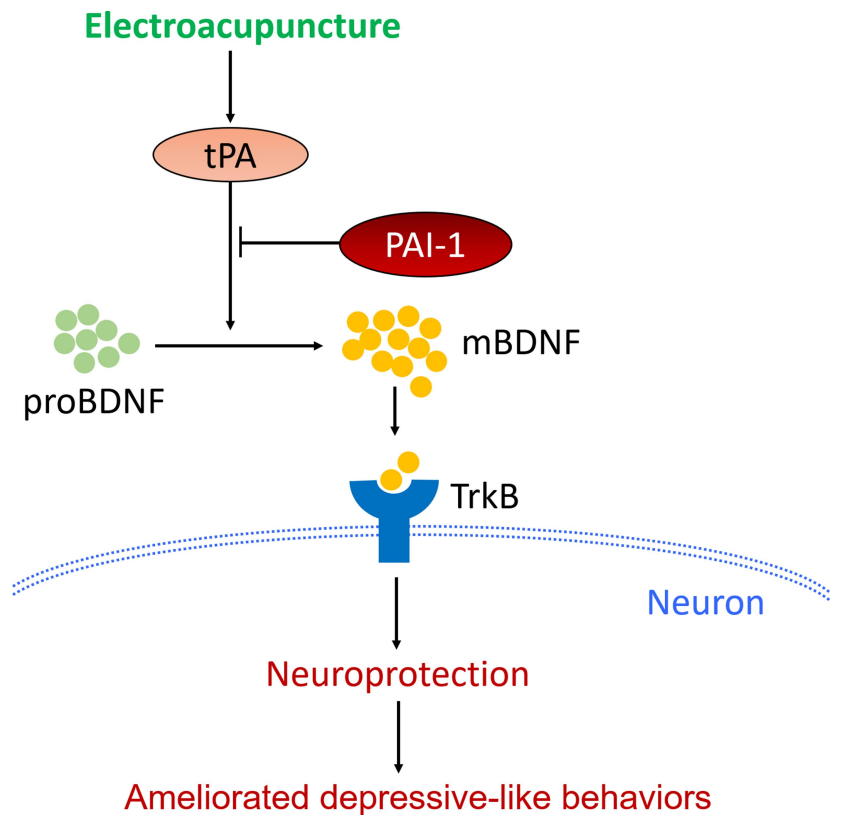

Figure 7 Schematic illustration of the potential mechanisms by which EA attenuated depressive-like behaviors in PSD models.

could be blocked by a specific tPA inhibitor PAI- $1 .^{36}$ This provided strong support for our conclusion that EA exerted its antidepressant effect at least partly through the activation of tPA pathway (Figure 7).

This study has several limitations. First, the activation of $\mathrm{tPA} / \mathrm{BDNF} / \mathrm{TrkB}$ pathway in the hippocampus had not been investigated, so the potential hippocampusrelated mechanism by which EA attenuated the depressive-like behaviors in PSD rats remains unclear. Second, only one inhibitor was used in this study, the inhibitors of BDNF and TrkB are also needed to further support our conclusion. Third, it is still not clear whether PAI-1 can produce a depressive-like phenotype. Our results has to be interpreted with caution given the uncertain role of PAI-1. Fourth, these findings must be interpreted with caution because the animal model of PSD cannot realistically simulate the pathophysiology involved in PSD patients.

\section{Conclusions}

In summary, this study demonstrated that EA ameliorated depressive-like behaviors in a rat model of PSD, and the improvement was achieved by promoting activation of the tPA/BDNF/TrkB pathway in the prefrontal cortex. The current results provided a new perspective on the mechanism by which EA attenuates the depressive-like behaviors following stroke. 


\section{Acknowledgments}

This work was supported by the Fundamental Research Funds for Central Public Welfare Research Institutes (No. 2018cz-3).

\section{Disclosure}

The authors report no conflicts of interest in this work.

\section{References}

1. Amani H, Habibey R, Shokri F, et al. Selenium nanoparticles for targeted stroke therapy through modulation of inflammatory and metabolic signaling. Sci Rep. 2019;9(1):6044. doi:10.1038/s41598019-42633-9

2. Lin DH, Zhang XR, Ye DQ, et al. The role of the two-pore domain potassium channel TREK-1 in the therapeutic effects of escitalopram in a rat model of poststroke depression. CNS Neurosci Ther. 2015;21 (6):504-512. doi:10.1111/cns.12384

3. Koo YS, Kim H, Park JH, et al. Indoleamine 2,3-dioxygenasedependent neurotoxic kynurenine metabolism contributes to poststroke depression induced in mice by ischemic stroke along with spatial restraint stress. Oxid Med Cell Longev. 2018;2018:2413841. doi:10.1155/2018/2413841

4. Yi ZM, Liu F, Zhai SD. Fluoxetine for the prophylaxis of poststroke depression in patients with stroke: a meta-analysis. Int J Clin Pract. 2010;64(9):1310-1317. doi:10.1111/j.1742-1241.2010.02437.x

5. Zhu J, Peng Q, Xu Y, et al. Morinda officinalis oligosaccharides ameliorate depressive-like behaviors in poststroke rats through upregulating GLUT3 to improve synaptic activity. FASEB J. 2020;34 (10):13376-13395. doi:10.1096/fj.201902546RR

6. Jin HJ, Pei L, Li YN, et al. Alleviative effects of fluoxetine on depressive-like behaviors by epigenetic regulation of BDNF gene transcription in mouse model of post-stroke depression. Sci Rep. 2017;7(1):14926. doi:10.1038/s41598-017-13929-5

7. Zhou Z, Lu T, Xu G, et al. Decreased serum brain-derived neurotrophic factor (BDNF) is associated with post-stroke depression but not with BDNF gene Val66Met polymorphism. Clin Chem Lab Med. 2011;49(2):185-189. doi:10.1515/CCLM.2011.039

8. Luo L, Li C, Du X, et al. Effect of aerobic exercise on BDNF/ proBDNF expression in the ischemic hippocampus and depression recovery of rats after stroke. Behav Brain Res. 2019;362:323-331. doi:10.1016/j.bbr.2018.11.037

9. Liang J, Yue Y, Jiang H, et al. Genetic variations in the p11/tPA/ BDNF pathway are associated with post stroke depression. $J$ Affect Disord. 2018;226:313-325. doi:10.1016/j.jad.2017.09.055

10. Zhou Z, Ding X, Yang Q, et al. Association between single-nucleotide polymorphisms of the tyrosine kinase receptor B (TrkB) and post-stroke depression in China. PLoS One. 2015;10 (12):e0144301. doi:10.1371/journal.pone.0144301

11. Luo L, Li C, Deng Y, et al. High-intensity interval training on neuroplasticity, balance between brain-derived neurotrophic factor and precursor brain-derived neurotrophic factor in poststroke depression rats. J Stroke Cerebrovasc Dis. 2019;28(3):672-682. doi:10.1016/j.jstrokecerebrovasdis.2018.11.009

12. Lv ZT, Shen LL, Zhu B, et al. Effects of intensity of electroacupuncture on chronic pain in patients with knee osteoarthritis: a randomized controlled trial. Arthritis Res Ther. 2019;21(1):120. doi:10.1186/ s13075-019-1899-6

13. Liu Z, Liu Y, Xu H, et al. Effect of electroacupuncture on urinary leakage among women with stress urinary incontinence: a randomized clinical trial. JAMA. 2017;317(24):2493-2501. doi:10.1001/jama.2017.7220
14. Liu Z, Yan S, Wu J, et al. Acupuncture for chronic severe functional constipation: a randomized trial. Ann Intern Med. 2016;165 (11):761-769. doi:10.7326/M15-3118

15. Zhao B, Li Z, Wang Y, et al. Manual or electroacupuncture as an add-on therapy to SSRIs for depression: a randomized controlled trial. J Psychiatr Res. 2019;114:24-33. doi:10.1016/j.jpsychires.2019.04.005

16. Wang Z, Dong H, Wang Q, et al. Effects of electroacupuncture on anxiety and depression in unmarried patients with polycystic ovarian syndrome: secondary analysis of a pilot randomised controlled trial. Acupunct Med. 2019;37(1):40-46. doi:10.1136/acupmed-2017011615

17. Amorim D, Amado J, Brito I, et al. Acupuncture and electroacupuncture for anxiety disorders: a systematic review of the clinical research. Complement Ther Clin Pract. 2018;31:31-37. doi:10.1016/j.ctcp.2018.01.008

18. Shen X, Xia J, Adams CE. Acupuncture for schizophrenia. Cochrane Database Syst Rev. 2014;(10):CD005475. doi:10.1002/14651858. CD005475.pub2

19. Li XB, Wang J, Xu AD, et al. Clinical effects and safety of electroacupuncture for the treatment of post-stroke depression: a systematic review and meta-analysis of randomised controlled trials. Acupunct Med. 2018;36(5):284-293. doi:10.1136/acupmed-2016-011300

20. Cai W, Ma W, Wang GT, et al. Antidepressant, anti-inflammatory, and antioxidant effects of electroacupuncture through sonic hedgehog-signaling pathway in a rat model of poststroke depression. Neuropsychiatr Dis Treat. 2019;15:1403-1411. doi:10.2147/NDT.S205033

21. Zhou CH, Xue F, Xue SS, et al. Electroacupuncture pretreatment ameliorates PTSD-like behaviors in rats by enhancing hippocampal neurogenesis via the Keap1/Nrf2 antioxidant signaling pathway. Front Cell Neurosci. 2019;13:275. doi:10.3389/fncel.2019.00275

22. Xue F, Xue SS, Liu L, et al. Early intervention with electroacupuncture prevents PTSD-like behaviors in rats through enhancing hippocampal endocannabinoid signaling. Prog Neuropsychopharmacol Biol Psychiatry. 2019;93:171-181. doi:10.1016/j.pnpbp.2019.03.018

23. Diaz-Ruiz A, Vacio-Adame P, Monroy-Noyola A, et al. Metallothionein-II inhibits lipid peroxidation and improves functional recovery after transient brain ischemia and reperfusion in rats. Oxid Med Cell Longev. 2014;2014:436429. doi:10.1155/2014/436429

24. Saha S, Hatch DJ, Hayden KM, et al. Appetite and weight loss symptoms in late-life depression predict dementia outcomes. $\mathrm{Am}$ $J \quad$ Geriatr Psychiatry. 2016;24(10):870-878. doi:10.1016/j. jagp.2016.05.004

25. Wang C, Wu C, Yan Z, et al. Ameliorative effect of Xiaoyao-jieyusan on post-stroke depression and its potential mechanisms. $J$ Nat Med. 2019;73(1):76-84. doi:10.1007/s11418-018-1243-5

26. Lim CM, Kim SW, Park JY, et al. Fluoxetine affords robust neuroprotection in the postischemic brain via its anti-inflammatory effect. J Neurosci Res. 2009;87(4):1037-1045. doi:10.1002/jnr.21899

27. Hu HM, Li B, Wang XD, et al. Fluoxetine is neuroprotective in early brain injury via its anti-inflammatory and anti-apoptotic effects in a rat experimental subarachnoid hemorrhage model. Neurosci Bull. 2018;34(6):951-962. doi:10.1007/s12264-018-0232-8

28. Kraeuter AK, Guest PC, Sarnyai Z. The open field test for measuring locomotor activity and anxiety-like behavior. Methods Mol Biol. 2019;1916:99-103. doi:10.1007/978-1-4939-8994-2_9

29. Li Y, Peng C, Guo X, et al. Expression of brain-derived neurotrophic factor and tyrosine kinase $\mathrm{b}$ in cerebellum of poststroke depression rat model. Chin Med $J$ (Engl). 2015;128(21):2926-2931. doi:10.4103/0366-6999.168058

30. Zhang E, Liao P. Brain-derived neurotrophic factor and post-stroke depression. J Neurosci Res. 2020;98(3):537-548. doi:10.1002/ jnr. 24510

31. Han Z, Wang Y, Qi L, et al. Differential association of serum bdnf with poststroke depression and poststroke anxiety. Arch Phys Med Rehabil. 2020;101(8):1355-1366. doi:10.1016/j.apmr.2020.04.011 
32. Zhang ZH, Wu LN, Song JG, et al. Correlations between cognitive impairment and brain-derived neurotrophic factor expression in the hippocampus of post-stroke depression rats. Mol Med Rep. 2012;6 (4):889-893. doi:10.3892/mmr.2012.1009

33. Egorova N, Cumming T, Shirbin C, et al. Lower cognitive control network connectivity in stroke participants with depressive features. Transl Psychiatry. 2018;7(11):4. doi:10.1038/s41398-017-0038-x

34. Marosi K, Mattson MP. BDNF mediates adaptive brain and body responses to energetic challenges. Trends Endocrinol Metab. 2014;25 (2):89-98. doi:10.1016/j.tem.2013.10.006
35. Haapasalo A, Sipola I, Larsson K, et al. Regulation of TRKB surface expression by brain-derived neurotrophic factor and truncated TRKB isoforms. J Biol Chem. 2002;277(45):43160-43167. doi:10.1074/jbc. M205202200

36. Zivkovic M, Starcevic Cizmarevic N, Lovrecic L, et al. The role of TPA I/D and PAI-1 4G/5G polymorphisms in multiple sclerosis. Dis Markers. 2014;2014:362708. doi:10.1155/2014/362708

\section{Publish your work in this journal}

Neuropsychiatric Disease and Treatment is an international, peerreviewed journal of clinical therapeutics and pharmacology focusing on concise rapid reporting of clinical or pre-clinical studies on a range of neuropsychiatric and neurological disorders. This journal is indexed on PubMed Central, the 'PsycINFO' database and CAS, and is the official journal of The International Neuropsychiatric Association (INA). The manuscript management system is completely online and includes a very quick and fair peer-review system, which is all easy to use. Visit http://www.dovepress.com/testimonials.php to read real quotes from published authors.

Submit your manuscript here: https://www.dovepress.com/neuropsychiatric-disease-and-treatment-journal 